\title{
SOSAP: A Pareto-efficient Spectrum Access Protocol for Cognitive Radio Networks
}

\author{
Stefano Iellamo \\ Foundation of Research and Technology Hellas \\ Institute of Computer Science, Greece \\ siellamo@ics.forth.gr
}

\author{
Marceau Coupechoux \\ LTCI, CNRS, Telecom ParisTech, \\ University Paris-Saclay, France \\ coupecho@enst.fr
}

\author{
Zaheer Khan \\ University of Oulu \\ CWC, Finland \\ zaheer.khan@ee.oulu.fi
}

\begin{abstract}
Decentralized cognitive radio networks (CRN) require efficient channel access protocols to enable cognitive secondary users (SUs) to access the primary channels in an opportunistic way without any coordination. In this paper, we develop a distributed spectrum access protocol for the case where the SUs aim to maximize the total system throughput while competing for spectrum resources. To model the competition amongst SUs, we formulate the spectrum access problem as a distributed welfare game, in which at each iteration each SU has to compute its marginal contribution to the system's welfare. Moreover, the SUs also need to decide which resource (channel) they should access at the next iteration. To address these challenges, we propose a stochastic learning algorithm based on payoff-based log-linear learning and prove its convergence towards a Pareto-efficient Nash equilibrium state.
\end{abstract}

\section{INTRODUCTION}

In decentralized cognitive radio networks (CRN), a fundamental while challenging task is the design of distributed spectrum (channel) access mechanisms enabling cognitive secondary users (SUs) to access the primary channels in an efficient way without any coordination. In this paper, we develop and analyze a spectrum access control mechanism where each user aims at maximizing the total system throughput. To this end, it follows a revision protocol based on payoff-based log-linear learning which will be shown to be able to orient the network towards a Pareto-efficient Pure Nash Equilibrium (PNE).

The problem of distributed spectrum access in CRNs has been widely addressed in the literature. A first set of papers assumes that the number of SUs is smaller than the number of channels. In this case, the problem is closely related to the classical Multi-Armed Bandit (MAB) problem [1]. Some works have investigated the issue of adapting traditional MAB approaches to the CRN context, among which Anandkumar et al. proposed two algorithms with logarithmic regret, where the number of SUs is known or estimated by each SU [2]. Complementary, other works assume large population of SUs and study the system dynamics under asymptotic assumptions. In [3], the authors propose a distributed learning procedure for spatial spectrum access which is proven to

S.Iellamo is supported by the European Union's Seventh Framework Programme (FP7/2007-2013) under grant agreement no 612361. M. Coupechoux is partly supported by the french ANR project NETLEARN ANR-13-INFR004. converge to a Nash Equilibrium (NE) in the asymptotic case. The analysis relies however on a random backoff mechanism, which requires the modification of the SUs packet structure for channel contention. In [4] the authors propose imitation rules that are used by a large population of SUs to converge to a Pure NE (PNE). In [4] it is also assumed that the SUs are able to capture packets transmitted by any other SU in the network. Differently from this literature, the spectrum access protocol proposed in this paper is proven to converge to a Pareto efficient payoff profile regardless of the number of SUs in the system. Furthermore, it is completely distributed and does not require any additional packet fields.

Other payoff-based algorithms have been proposed in the literature. In [5] and [3] for instance, the authors develop payoff-based mechanisms that are proven to converge to a PNE of the formulated game. In those cases however, the best PNE is not Pareto-efficient and, furthermore, no equilibrium selection is performed.

The unique properties possessed by payoff-based log-linear learning (PLLL) have been firstly studied by Marden [6], and then readily applied by a handful of works (see the recent [7] and references therein). To the best of our knowledge, we are the first to use PLLL to attain Pareto-efficient PNE in the context of cognitive radio networks. Our contribution in this paper is therefore three-fold:

- We formulate the spectrum access coordination problem as a distributed welfare game (Section III)

- We propose a distributed learning algorithm based on PLLL and show its convergence towards a Pareto-efficient PNE for the considered model (Section IV).

- We analyze the effectiveness of our solution by means of an extensive numerical analysis with real settings (Section VI).

\section{System Model}

In this paper, we consider the downlink of a primary network and a set of SUs which are allowed to opportunistically access the primary network spectrum (see Fig. 1, where we illustrate an example of considered primary and secondary network). The primary spectrum consists of a set $\mathcal{C}$ of $C$ frequency channels, each with bandwidth $B$. The users in the primary network operate in a synchronous time-slotted 


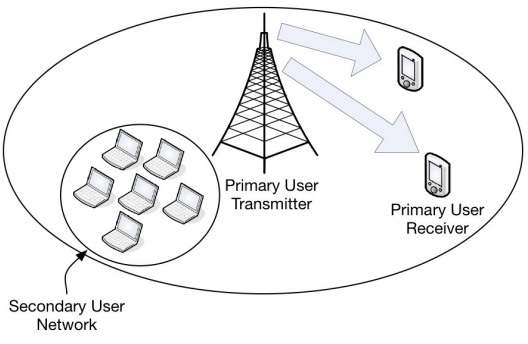

Fig. 1. Network model.

fashion. At each PU time slot, channel $i$ is free with probability $\mu_{i}$, where such probabilities are assumed to be i.i.d. with regard to time slot and considered channel. A set $\mathcal{N}$ of $N$ SUs tries to opportunistically access the channels when they are left free by the Primary Users (PUs).

Let us define an iteration $t$ as a period of time during which channel switches by SUs are not allowed. This means, a SU's channel selection remains fixed throughout an iteration $t$. A schematic representation of the resulting block structure is shown in Fig. 2.

In our model, each SU $j$ is modeled as an agent aiming at maximizing the total system throughput. To this end, SUs implement a generic MAC protocol (such as, e.g., CSMA/CA) to avoid collisions throughout an iteration $t$.

At the end of each iteration, each SU has to decide the strategy for the next iteration, i.e., which channel will be accessed throughout next iteration $t+1$.

Let $s_{j}(t)$ denote $\mathrm{SU} j$ 's accessed channel at the iteration $t$. We define an allocation $\mathbf{s}$ as the set of all strategy profiles $\left\{s_{j}(t)\right\}$ for all $j \in \mathcal{N}$. The instantaneous throughput an SU $j$ can achieve on channel $i$ in terms of packets per second, denoted as $T_{j i}$, can be expressed as a function of $\mu_{i}$ and $\{\mathbf{s}\}_{i}$, where $\{\mathbf{s}\}_{i}$ is the set of contending SUs using channel $i$ in allocation $\mathbf{s}$. The expected value of $T_{j i}$, which has to be intended as the long-term throughput when $t$ is large, can be written as: $\mathbb{E}\left[T_{j i}\right]=f_{j i}\left(\mu_{i},\{\mathbf{s}\}_{i}\right)$. In this paper, SUs implement a generic random access protocol to avoid collisions. Thus, $f_{j i}($.$) can take the following$ form: $f_{j i}()=.B \mu_{i} p_{j}\left(\{\mathbf{s}\}_{i}\right)$ where $p_{j}\left(\{\mathbf{s}\}_{i}\right)$ is a decreasing user-specific function denoting the successful transmission probability for $\mathrm{SU} j$ in allocation $\{\mathbf{s}\}_{i}$ (please recall that the number of SUs operating on a given channel $i$ is fixed throughout an iteration $t$ ). $B$ is a constant standing for the available bandwidth per channel. Without loss of generality, we will now assume that $B=1$.

\section{GAME FORMULATION}

Let us express the total throughput of the system in the form of separable welfare functions [8]:

$$
W(\mathbf{s})=\sum_{i \in \mathcal{C}, j \in \mathcal{N}} f_{j i}(.)=\sum_{i \in \mathcal{C}} W_{i}\left(\{\mathbf{s}\}_{i}\right)
$$

Thus, the welfare $W_{i}\left(\{\mathbf{s}\}_{i}\right)$ linked to a given channel $i$ can be thought as the total (cumulative) secondary throughput

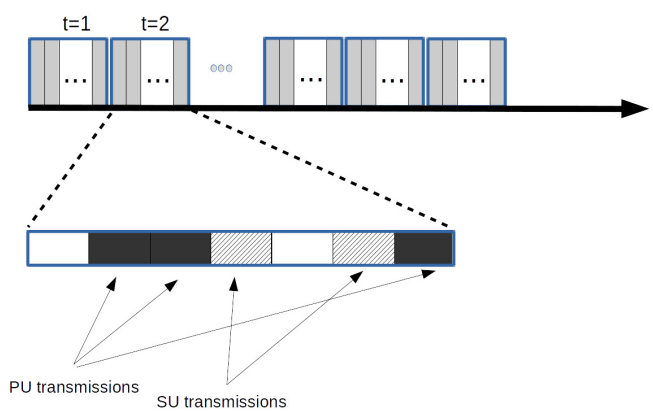

Fig. 2. Representation of the blocks structure utilized by the SUs.

available at $i$ when the set $\{\mathbf{s}\}_{i}$ of SUs is operating on it. For a given MAC random access scheme, such value depends on the load conditions of the contending stations [9], [10].

In a distributed welfare game, each agent's utility is defined as some fraction of the welfare generated by the resource the agent is using. More formally, the utility of agent $j$ playing strategy $s_{j} \in \mathcal{C}$ for a given allocation $\mathbf{s}:=\left(s_{j}, \mathbf{s}_{-j}\right)$ at iteration $t$ is given by:

$$
U_{j}(t)=\pi_{s_{j}}\left(\{\mathbf{s}\}_{s_{j}}, t\right)
$$

where $\pi_{s_{j}}: \mathcal{N} \times 2^{\mathcal{N}} \rightarrow \mathbb{R}$ is referred to as the distribution rule at resource $s_{j}$. Note that in order to lighten notation we will some time omit the iteration index if the latter refers to the current iteration $t$.

In our case, we adopt the marginal contribution distribution rule:

$$
\pi_{s_{j}}^{M C}\left(\{\mathbf{s}\}_{s_{j}}\right)=W_{s_{j}}\left(\{\mathbf{s}\}_{s_{j}}\right)-W_{s_{j}}\left(\{\mathbf{s}\}_{s_{j}} \backslash\{j\}\right)
$$

which can be interpreted as a user's contribution to the total experienced welfare. In this way, the utility for each player $j$ on channel $i$ can be approximated as follows:

$$
U_{j} \simeq \tilde{U}_{j}=\frac{\xi_{j i}-c_{j i}}{n_{t}}
$$

where $n_{t}$ is the number of time slots in one iteration $t, \xi_{j i}$ and $c_{j i}$ are the total number of j's correct transmissions and collisions on channel $i$ respectively. As one can easily infer from (4) the marginal contribution of a single player to the total system throughput is positive iff its correct transmissions outnumber the collisions caused. Note that the value obtained by means of the formula above is a lower bound of the real value (i.e., $\tilde{U}_{j} \leqslant U_{j}$ ), as collisions with more than 2 terminals involved should not be subtracted (intuitively, collision would have occurred anyway if one of the 3 or more stations involved had stepped back from transmitting). However, the presented formula suits our distributed scenario (can be obtained through own transmission statistics and sensing operations while not transmitting); Moreover, the approximation is accurate if the probability of multiple collisions is sufficiently low. This holds true for loads not exceeding too much the random access protocol saturation point. 
We are now in the condition of defining the game $\mathcal{G}$ more formally.

Definition 1 (Channel allocation game $\mathcal{G})$. The channel allocation game $\mathcal{G}$ is a 3-tuple $\left(\mathcal{N}, \mathcal{C},\left\{U_{j}\right\}\right)$. The strategy $s_{j} \in \mathcal{C}$ is the channel that $S U j$ accesses at the current iteration.

Once each player $j$ has chosen its strategy $s_{j}$, its playerspecific utility function $U_{j}$ is defined as

$$
U_{j}=\pi_{s_{j}}^{M C}\left(\{\mathbf{s}\}_{s_{j}}\right)
$$

We know from [8] that $\mathcal{G}$ possesses at least one PNE (distributing the welfare as in (3) results in a potential game with potential $W$, meaning that every distributed welfare game with decreasing marginal contributions is a potential game). We thus need to come up with a mechanism that is able to find out the best PNE, i.e., the equilibrium point where global welfare is maximized. To this end, we use payoff-based log-linear learning [6], which is proven to converge towards such desirable equilibrium point.

\section{SOSAP: A SPECTRUm ACCESS PRotocol BASEd ON PAYOFF-BASED LOG-LINEAR LEARNING}

We now provide details of the designed spectrum access scheme, termed as SOSAP (Socially Optimal Spectrum Access Protocol).

SOSAP (shown in Algorithm 1) is based on Payoff-based Log-Linear Learning (PLLL), which has been proposed by Marden in [6] and can be seen as the distributed version of the more famous Logit-response rule ([11], [12]). It works as follows:

- Throughout an iteration $t$, no channel switches are allowed and each SU $j$ accesses the chosen channel $i$ according to the random access protocol associated to $i$.

- At the end of each iteration $t, j$ calculates its own utility $U_{j}(t)$, which in our case is the marginal contribution to the total offered system throughput. In SOSAP, $j$ calculates its approximate utility $\tilde{U}_{j}$ according to (4).

- The strategy for the next iteration $t+1$ is chosen according to the payoff-based log-linear rule with probability $1-\epsilon(t)$ and uniformly randomly (this event is referred to as a mutation or tremble in the literature of evolutionary models with noise) with probability $\epsilon(t)$, where $\epsilon(t)=\left(e^{-1 / \tau(t)}\right)^{m}, \tau(t)>0$ is the algorithm temperature and $m$ a large enough constant.

\section{Convergence Analysis}

For the sake of a self-contained presentation, let us firstly provide a few definitions and a couple of propositions before stating the main theorem.

Definition 2 (Pure Nash Equilibrium). A Pure Nash Equilibrium (PNE) is a point $\mathbf{s}^{*}$ in the action profiles space, from which no user has incentive to deviate unilaterally. The strategy profiles at a PNE can be defined as follows:

$$
\mathbf{s}^{*} \triangleq \underset{s_{j} \subseteq C}{\operatorname{argmax}} U_{j}\left(s_{j}, \mathbf{s}_{-j}^{*}\right), \quad \forall j \in \mathcal{N} .
$$

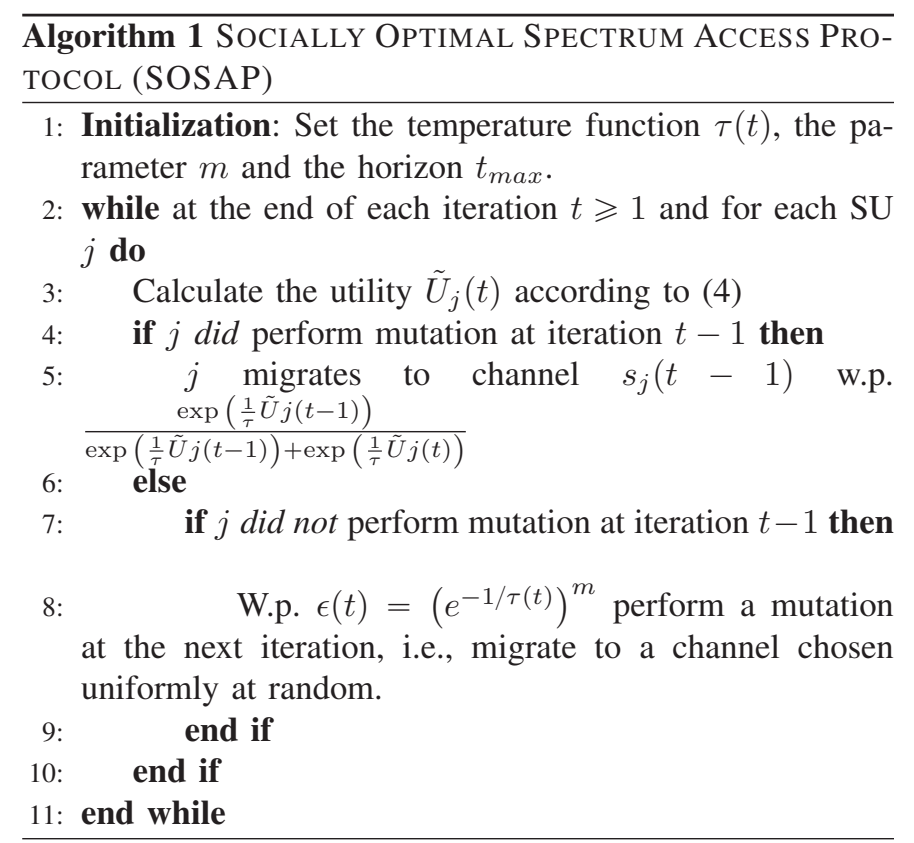

where $\mathbf{s}_{-j}=\left\{s_{1}, . ., s_{j-1}, s_{j+1}, \ldots, s_{C}\right\}$ is the collection of channels chosen by all users except user $j$.

Definition 3 (Pareto-efficient allocation). In a distributed welfare game, a Pareto-efficient allocation $\mathbf{s}^{o}$ is a point in the action profile space where the system global welfare is maximized. That is, $\mathbf{s}^{o} \in \operatorname{argmax}_{\mathbf{s}} W(\mathbf{s})$.

Definition 4 (Price of Stability and Price of Anarchy). For a generic game $G$, let $\mathcal{E}$ denote the set of equilibria and let PoS and PoA denote the Price of Stability and Price of Anarchy respectively.

$\operatorname{PoS}(G)$ is then the ratio between the best objective function value of one of its equilibria and that of an optimal outcome for the considered game $G$.

$$
\operatorname{PoS}(G):=\max _{\mathbf{s} \in \mathcal{E}(G)} \frac{W\left(\mathbf{s}^{*}\right)}{W\left(\mathbf{s}^{o}\right)}
$$

Similarly, $\operatorname{PoA}(G)$ is defined as the ratio between the worst objective function value of one of its equilibria and that of an optimal outcome for the considered game $G$.

$$
\operatorname{PoA}(G):=\min _{\mathbf{s} * \mathcal{E}(G)} \frac{W\left(\mathbf{s}^{*}\right)}{W\left(\mathbf{s}^{o}\right)}
$$

Proposition 1 ([6], [8]). In a distributed welfare game where the players' utility functions are user-specific and decreasing in the number of contending stations, there always exists at least one Pure Nash Equilibrium and payoff-based log-linear learning converges to the best PNE of the game.

Proposition 2 ([8]). The Price of Stability (PoS) and Price of Anarchy (PoA) af a distributed welfare game characterized by players' utility function in the form of marginal contributions are equal to 1 and $1 / 2$ respectively.

In other words, the best PNE is on the Pareto frontier and is therefore Pareto-efficient. The worst PNE is worth half of the best one. 
We can write the following important result:

Theorem 1. For the distributed welfare game $\mathcal{G}$, if 1) the players' marginal contributions are inversely proportional to the congestion level, 2) all SUs adopt at each iteration $t$ Payoff-based Log-Linear Learning as revision scheme, and 3) $\epsilon(t)=\left(e^{-1 / \tau(t)}\right)^{m}$ with $m$ sufficiently large, then the system dynamics converge a.s. to a Pareto-efficient Pure Nash equilibrium $\mathbf{s}^{\circledast}$ as the temperature $\tau(t)$ vanishes.

Proof. It follows from Proposition 1, Proposition 2 and Theorem 6.1 in [6].

Remark: PLLL was recently proven to converge when players' utility estimations are noise corrupted [13] and, more recently, to be efficient even over stochastic communication links [14]. As a main constraint, the distribution of the estimation error should be 0 -mean, meaning that the agents are supposed to be equipped with an unbiased estimator of the true utility. In our case, the estimation error depends on approximation (4) and iteration duration. Therefore, the estimator is normally biased. However, if the number of SUs is sufficiently small wrt to the number of channels and the iteration duration is sufficiently large, such error is intuitively very small and can be arguably neglected. We will show by simulation (in Section VI) that this is indeed the case for the considered scenario.

\section{Simulation ANAlysis}

For the simulation we consider a cognitive radio network, made of 10 SUs and 3 channels characterized by availabilities $\mu=\left[\begin{array}{lll}0.1 & 0.7 & 0.8\end{array}\right]$. We consider a complete interference graph and saturation SU operation conditions. We let the SUs access the chosen channel by means of CSMA/CA with RTS/CTS. Protocol and CR parameters are taken according to [15] and [16] respectively (for space reason we do not report them). We assume perfect sensing at the SUs, i.e., any transmission of any PU on a channel is perfectly sensed by SUs sensing that channel and thus no collision occurs between PUs and SUs. The duration of one PU time slot is fixed. A small initial part of it, of fixed length as well, is used by the SUs for sensing the presence of the PU. If a packet is partially transmitted at the end of the PU time slot, the transmission is reactivated, without loss of information, at the first PU-slot which is sensed non-occupied by the PU. Each channel offered secondary throughput (i.e., without considering the PU activity) as a function of the number of contending SUs is depicted in Fig 3. We assume that iterations are long enough so that the throughput obtained by the contending stations equalize at the end of an iteration in all channels.

At the end of each iteration, the SUs calculate their utility according to Eq. (4) and revise their strategy according to SOSAP (Algorithm 1). For performance comparison, we choose RSAP (which stands for Retrospective Spectrum Access Protocol), the payoff-based algorithm proposed in [5], where the SUs utility function is associated to their expected throughput and the equilibria are characterized by

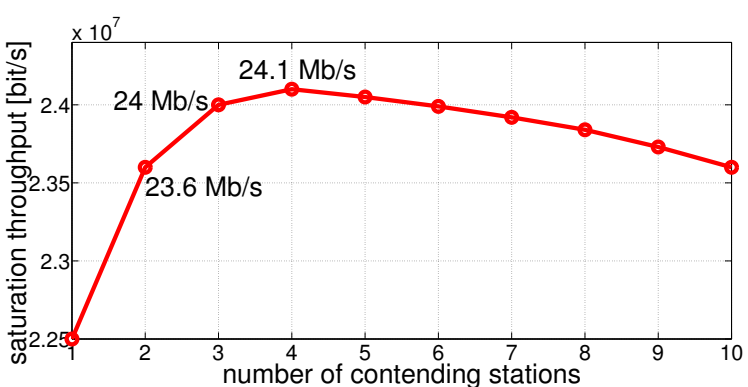

Fig. 3. Saturation throughput as a function of the number of the contending stations operating on the same channel. All channels deliver the largest amount of throughput when there are $n_{j}=4$ contending SUs on them; the throughput starts to decrease afterwards.

high fairness.

Fig. 4 and Fig. 5 display respectively one realization and an average over 200 independent realizations of the secondary cumulative throughput as a function of the iteration $t$. In Fig. 4 we clearly see that as the algorithm temperature decreases SOSAP converges towards the basin of attraction of the Pareto-efficient allocation. The latter is characterized by 4 SUs on channel 2, 4 SUs on channel 3 and 2 SUs on channel 1. The cumulative SU throughput at the Pareto-efficient PNE can be calculated as $10^{6} \cdot[0.8 \cdot 24.1+0.7 \cdot 24.1+0.1 \cdot 23.6]=38.51 \mathrm{Mb} / \mathrm{s}$, where $0.8,0.7$ and 0.1 are the channel availabilities in channel 3,2 and 1 respectively and the cumulative SU throughput values are drawn from Fig. 3.

In Fig. 5 we compare the average behavior of SOSAP and RSAP. We use the same temperature functions for both algorithms. The figure shows that SOSAP clearly outperforms RSAP in terms of cumulative SU throughput: For the considered network, SOSAP cumulative secondary throughput is in average $7 \%$ higher than that achieved by RSAP. Furthermore, SOSAP always converges to a Paretooptimal solution, while RSAP cumulative SU throughput even shows a decreasing trend in the displayed iteration window.

Fig. 6 shows the performance achieved by SOSAP and RSAP in terms of Jain's fairness index, which reaches the maximum of 1 when the resource (the throughput in our case) is equally shared amongst users [17]. Looking at the figure, we see that RSAP is clearly more fair than SOSAP. This means, at the NE the SUs will achieve approximately the same throughput with RSAP, while this is not the case with SOSAP. This is because RSAP equilibria are budget-balanced (i.e., they feature a high fairness) but not Pareto-efficient (for the game formulated in [5] $\mathrm{PoA}=\mathrm{PoS}=1 / 2$ ).

\section{DISCUSSION}

From the previous sections, it seems that SOSAP and RSAP go after different objectives; social optimality and fairness respectively. Thus, one might wonder: Is it possible to design a distributed learning algorithm achieving both social and 


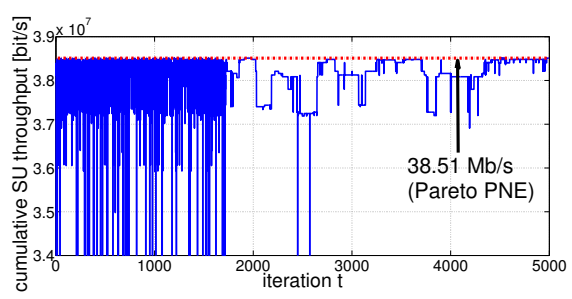

Fig. 4. Performance of SOSAP in terms of aggregate secondary throughput as a function of the iteration $\mathrm{t}$ (one realization).

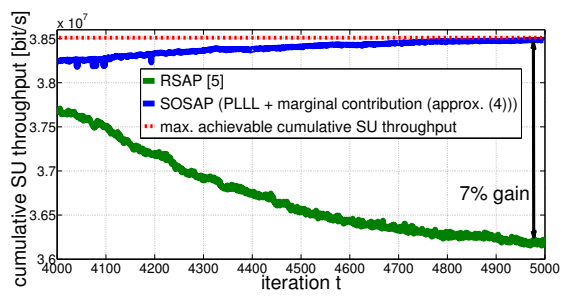

Fig. 5. SOSAP and RSAP [5] secondary throughput comparison as a function of the iteration (average).

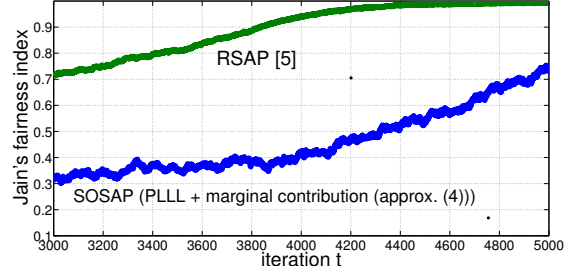

Fig. 6. SOSAP and RSAP fairness comparison as a function of the iteration (average). fairness optimality? Although we don't have a direct answer to this question, we argue that social optimality might be a more reasonable objective to pursue in some particular cases:

- The system is fair by nature: think for example of a scenario where a network of identical secondary users has to coordinate to access a set of identical ISM bands (i.e., without PUs) by means of the same spectrum access protocol (e.g., CSMA/CA). In this case, SOSAP will be able to achieve both high fairness and social optimality.

- The system is made of heterogeneous SUs (with different load conditions). In other words, the throughput obtained by each SU depends on the identity of the SUs on the same channel. In this particular case a PNE is not guaranteed to exist for the game formulated in [5] (insights on PNE existence in congestion games can be found in [18] and [19]).

- The system includes battery-powered devices (e.g., IoT nodes): as one can infer from Eq. (4), SOSAP minimizes the number of collisions and thereby is preferable from an energy consumption perspective.

\section{CONCLUSION}

In this paper we have studied the problem of distributed spectrum access in cognitive radio network, by focusing on system throughput maximization. We have developed a distributed spectrum access revision scheme based on payoffbased log-linear learning and have shown its convergence towards the best Nash Equilibrium of the designed game, which is also Pareto-efficient. We have shown how this comes at the cost of a lower fairness among the SUs when compared to the case where the SUs associate their expected throughput to their utilities. Extensions involving multi-antenna users as well as channel bonding/aggregation are interesting research subjects which are left for future work.

\section{REFERENCES}

[1] A. Mahajan and D. Teneketzis. Foundations and Applications of Sensor Management, chapter Multi-armed Bandit Problems, pages 121-151. Springer-Verlag, 2007.

[2] A. Anandkumar, N. Michael, and A. Tang. Opportunistic Spectrum Access with Multiple Users: Learning under Competition. In Proc. IEEE International Conference on Computer Communication (INFOCOM), San Diego, CA, Apr. 2010.

[3] Xu Chen and Jianwei Huang. Spatial spectrum access game: nash equilibria and distributed learning. In Proceedings of the thirteenth ACM international symposium on Mobile Ad Hoc Networking and Computing, MobiHoc '12, pages 205-214, New York, NY, USA, 2012. ACM.
[4] Stefano Iellamo, Lin Chen, and Marceau Coupechoux. Proportional and double imitation rules for spectrum access in cognitive radio networks. Computer Networks, 2013.

[5] S. Iellamo, Lin Chen, and M. Coupechoux. Retrospective spectrum access protocol: A payoff-based learning algorithm for cognitive radio networks. In 2014 IEEE International Conference on Communications (ICC), pages 1422-1427, June 2014.

[6] J.R. Marden and J.S. Shamma. Revisiting log-linear learning: Asynchrony, completeness and payoff-based implementation. In 2010 48th Annual Allerton Conference on Communication, Control, and Computing (Allerton), pages 1171-1172, September 2010.

[7] M.S. Ali, P. Coucheney, and M. Coupechoux. Load balancing in heterogeneous networks based on distributed learning in potential games. In 2015 13th International Symposium on Modeling and Optimization in Mobile, Ad Hoc, and Wireless Networks (WiOpt), pages 371-378, May 2015.

[8] Jason R. Marden and Adam Wierman. Distributed Welfare Games. Operations Research, 61(1):155-168, February 2013.

[9] G. Bianchi, L. Fratta, and M. Oliveri. Performance evaluation and enhancement of the CSMA/CA MAC protocol for 802.11 wireless LANs. In, Seventh IEEE International Symposium on Personal, Indoor and Mobile Radio Communications, 1996. PIMRC'96, volume 2, pages 392-396 vol.2, October 1996.

[10] Rama Krishna Challa, Saswat Chakrabarti, and Debasish Datta. An Improved Analytical Model for IEEE 802.11 Distributed Coordination Function under Finite Load. International Journal of Communications, Network and System Sciences, 02(03):237-247, 2009.

[11] Lawrence E. Blume. The Statistical Mechanics of Strategic Interaction. Games and Economic Behavior, 5(3):387-424, July 1993.

[12] Carlos Alós-Ferrer and Nick Netzer. The logit-response dynamics. Games and Economic Behavior, 68(2):413-427, March 2010.

[13] D.S. Leslie and J.R. Marden. Equilibrium selection in potential games with noisy rewards. In 2011 5th International Conference on Network Games, Control and Optimization (NetGCooP), pages 1-4, October 2011.

[14] Arjun Muralidharan, Yuan Yan, and Yasamin Mostofi. Binary Log-Linear Learning with Stochastic Communication Links. arXiv:1412.4166 [cs], December 2014. arXiv: 1412.4166.

[15] B. Mawlawi, J.-B. Dore, N. Lebedev, and J.-M. Gorce. Multiband CSMA/CA with RTS-CTS strategy. In 2014 IEEE 10th International Conference on Wireless and Mobile Computing, Networking and Communications (WiMob), pages 628-633, October 2014.

[16] S. Iellamo, L. Chen, and M. Coupechoux. Imitation-based Spectrum Access Policy for CSMA/CA-based Cognitive Radio Networks. In Proc. IEEE Wireless Communications and Networking Conference (WCNC), Paris, France, April 2012.

[17] R. Jain, D. Chiu, and W. Hawe. A Quantitative Measure of Fairness and Discrimination for Resource Allocation in Shared Computer Systems. Research Report TR-301, DEC, 1984.

[18] Igal Milchtaich. Congestion Games with Player-Specific Payoff Functions. Games and Economic Behavior, 13(1):111-124, March 1996.

[19] Gürdal Arslan, Jason R. Marden, and Jeff S. Shamma. Autonomous vehicle-target assignment: a game theoretical formulation. ASME JOURNAL OF DYNAMIC SYSTEMS, MEASUREMENT AND CONTROL, page 2007, 2007. 